\title{
INNOVACIONES TECNOLÓGICAS INCORPORADAS EN LA COMUNIDAD DE REGANTES DE L'ALCÚDIA (VALENCIA)
}

\author{
Carbonell Solbes N. ${ }^{1}$, Pardo Cuenca, Coral ${ }^{2}$
}

\author{
${ }^{1}$ Ingeniero Superior de Proyectos, Obra y Explotación de la Oficina de Valencia de SEIASA, \\ E-mail: n.carbonell@seiasa.es.
}

${ }^{2}$ Técnico Medio de Obras del Grupo TRAGSA en la Gerencia de Valencia, E-mail: cpardo1@tragsa.es

\section{Resumen}

Este trabajo pretende transmitir cómo las obras de "MEJORA DEL SISTEMA DE RIEGO EN EL SECTOR II - FASE I Y FASE II, L'ALCÚDIA, DE LA ZONA REGABLE DEL CANAL JÚCAR - TURIA (VALENCIA)" han contribuido a la optimización de los regadíos de la zona incorporando innovaciones tecnológicas fruto del esfuerzo conjunto entre Ministerio de Agricultura, SEIASA, Consellería de Presidencia y Agricultura, Pesca, Alimentación y Agua, la propia Comunidad de Regantes y Tragsa. Estas innovaciones incorporadas en dichas obras se clasifican en las siguientes áreas:

- Innovación en hidrante multiusuario.

- Sistema de monitorización ambiental multipropósito.

- Mejora de la gestión integral hídrica y energética.

- Mejora medioambiental.

- Mejora de calidad de las aguas y seguridad en abastecimiento hídrico en las Comunidades de Regantes en periodos de sequía.

\section{1- Introducción y objetivos}

El 13 de noviembre de 1985 se constituye la Comunidad General de Usuarios del Canal Júcar-Turia. Por orden de la Conselleria de Medio Ambiente de fecha de 9 de abril de 1.996 se aprueba el Plan de Obras de Interés General para la modernización del regadío de la zona regable del Canal Júcar-Turia. La Comunidad General, a través de sus Comunidades de base, se ha caracterizado por la decisión de acometer de forma continuada dicho Plan de Obras.

En el momento actual, el Canal Júcar-Turia, constituye el eje de una amplia zona de regadíos de $\mathbf{2 5 . 0 0 0}$ has, con un número aproximado de $\mathbf{3 5 . 0 0 0}$ usuarios. Dichos usuarios forman parte de diferentes comunidades de regantes, siendo 7 las Comunidades de la Subzona Margen Izquierda y de 14 Comunidades de Regantes pertenecientes a la Subzona Margen Derecha.

Sus regantes poseen un perfil innovador y, por tanto, aúnan esfuerzos para impulsar nuevos cultivos que proporcionen riqueza y estabilidad. No es posible mencionar el Canal Júcar Turia sin destacar la revolución que el Caqui ha trasladado a su zona regable, con la proyección comercial que la Denominación de Origen "KAKI Ribera del Xúquer" ha posibilitado. Con el fin de seguir desarrollando cultivos competitivos se puede observar 
como los agricultores de la zona prueban cambios varietales, principalmente en frutales de hueso (albaricoque, melocotón, paraguayo, nectarina...) y realizan importantes inversiones.

Hoy en día, gracias a la decisión unánime de mejorar la competitividad de sus cultivos, los agricultores, con ayuda de Instituciones Públicas y Privadas, han posibilitado la realización de nuevas inversiones para mejorar sus regadíos, con una tendencia clara hacia la tecnificación y profesionalización en la gestión técnica y administrativa.

En la modernización de regadíos se aplican las tecnologías y recursos adaptados a cada zona para lograr una mayor eficacia en el riego repercutiendo significativamente en una mejora en la gestión y uso eficiente del agua.

Este trabajo pretende transmitir cómo las obras de "MEJORA DEL SISTEMA DE RIEGO EN EL SECTOR II - FASE I Y FASE II, L'ALCÚDIA, DE LA ZONA REGABLE DEL CANAL JÚCAR - TURIA (VALENCIA)" han contribuido a la optimización de los regadíos de la zona incorporando innovaciones tecnológicas fruto del esfuerzo conjunto entre Ministerio de Agricultura, SEIASA, Consellería de Presidencia y Agricultura, Pesca, Alimentación y Agua, la propia Comunidad de Regantes y Tragsa.

Como antecedentes indicaremos que en 2003 fue redactado el proyecto de Mejora del Sector II, L’Alcúdia, el cual, fue distribuido en dos fases, dos obras independientes.

El Sector II - L'Alcúdia se encuentra en el margen derecho del Canal Júcar- Turia, y está delimitado por la Acequia Real del Júcar, la Riera de la Dehesa, la Riera del Saboner, el T.M. de Benimodo, la Riera de Matamoros, el Canal Júcar- Turia y la Rambla de la Señora hasta la Acequia Real del Júcar.

El sistema utilizado del riego antes de la modernización era en superficie (o a manta), de manera que existía una red extensa de acequias que partiendo de las tomas efectuadas en el Canal distribuían el agua a todo el sector.

El promotor de la fase I fue el Ministerio de Agricultura comenzando en mayo de 2007 y finalizando en Abril de 2012. Esta obra de la fase I de l'Alcúdia comprendió los elementos de cabecera (depósitos de almacenamiento, estaciones de bombeo, cabezales de filtrado y fertirrigación e instalaciones eléctricas) y la red principal.

La segunda fase de la obra fue realizada entre 2012 y 2014 mediante el convenio entre SEIASA, la Comunidad de Regantes Sector II La Garrofera-L'Alcúdia y la Consellería de Presidencia y Agricultura, Pesca, Alimentación y Agua, comprendiendo la terminación de la red secundaria y completando la totalidad de la modernización del regadío para su uso por el usurario (red terciaria, hidrantes, sistema de telecontrol, asfaltado de caminos, construcción de una nueva estación de bombeo, etc.) Fase II.

La empresa Tragsa fue la encargada de ejecutar toda la obra, tanto la Fase I como la

Las infraestructuras realizadas con la transformación al riego localizado abastecen una superficie regable de 813 ha, cítricos mayoritariamente y caqui, siendo 978 los regantes beneficiarios dentro de los T.T.M.M. de l'Alcúdia, Alzira y Guadassuar.

Esta obra, esfuerzo conjunto de todos los Organismos e Instituciones implicadas así como de la propia Comunidad de Regantes, ha permitido la optimización de los regadíos de la zona desarrollando y aplicando innovaciones tecnológicas. 
Las exigencias en las obras de modernización de regadíos se han incrementado en los últimos años debido a:

- Una elevada competitividad en el mercado que requiere una alta eficiencia en la producción así como una necesaria optimización de los costes (en los que se incluye los gastos de agua, energía, fertirrigación y mano de obra en explotación).

- La necesidad de facilitar el manejo, gestión y explotación del riego, mediante herramientas informativas que mejoren la calidad de vida del regante.

- Los continuos incrementos en el precio de la energía y variaciones de las tarifas eléctricas según las distintas épocas del año y horarios.

- La alta incidencia de los robos en los equipamientos de riego en el campo.

- La obligación de alertar y reducir los daños ocasionados por los agentes meteorológicos.

Por tanto, en las actuaciones realizadas en la Comunidad de Regantes de L'Alcúdia se han incorporado nuevos avances tecnológicos obteniendo la modernización de los regadíos exigida en la actualidad.

\section{2- Mejoras e innovaciones tecnológicas aplicadas a la modernización de regadíos.}

La aportación a la innovación tecnológica que se presenta se puede clasificar en las siguientes áreas:

\section{- Innovación en hidrante multiusuario}

Se implanta una novedosa tipología de hidrante multiusuario "Tipo Costella" (Figura 1) con una serie de ventajas, destacando los colectores de material plástico.

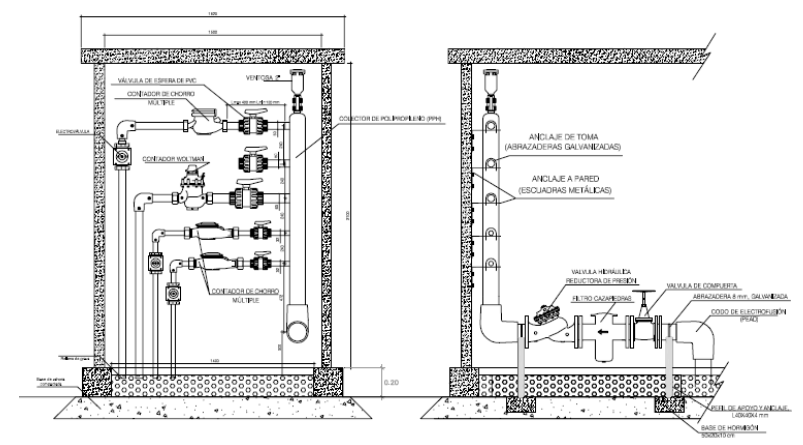

Figura 1. Esquema hidrante multiusuario "Tipo Costella"

La definición de colector establecido en la UNE 53943:2009 indica que es un elemento compuesto por un tubo vertical u horizontal, que alimenta los contadores divisionarios sirviendo de soporte a dichos aparatos y a sus válvulas. 
Dentro de la toma de decisiones relevantes en la vida útil de las instalaciones sobre el equipamiento a instalar es la correcta selección de los materiales en virtud del uso que vaya a hacerse del mismo, de la ubicación, del mantenimiento que se vaya a realizar y de la calidad de agua que vaya a emplearse en el riego.

El colector del hidrante de riego es un elemento que tradicionalmente ha sido ejecutado con materiales metálicos, acero al carbono con tratamientos o no de galvanizado o pintura epoxi. Hoy en día, con la incorporación en los sistemas de riego de aguas con productos químicos (para fertirrigación o limpieza de tuberías), de aguas residuales o salinas, o bien aguas de diversos orígenes con alto grado de poder de oxidación, ha provocado que se busquen alternativas a estos materiales metálicos.

Al ser el colector la pieza clave del hidrante debe ser sometido a unos procesos adecuados de calidad que garanticen su estanqueidad y una resistencia mecánica adecuada, para ello, en función de las características de la instalación se ha de determinar cuál es el mejor material plástico.

En cuanto a los materiales plásticos más utilizados en colectores para hidrantes de riego encontramos el polietileno de alta densidad (PP) y el polipropileno (PPR o PPH) (Figura 2) que presentan características diferentes entre sí, siendo las más notorias la rigidez y el coeficiente de dilatación las cuales confieren características diferentes a ambos materiales.

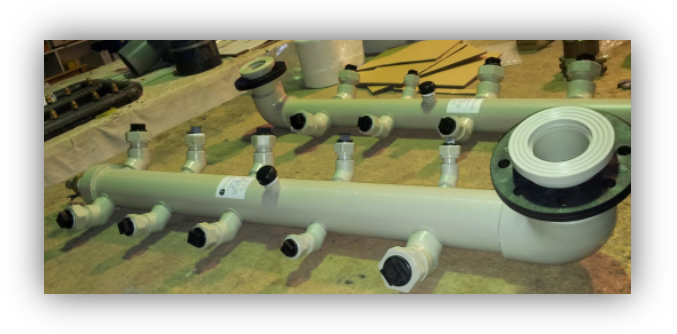

Figura 2. Colector de polipropileno. CCRR L'Alcúdia

La selección de materiales plásticos en colectores de hidrantes de riego ha sido principalmente debido a que los colectores metálicos, por causas diversas, han presentado en alguna ocasión oxidaciones importantes con difícil solución técnica.

Los colectores plásticos para hidrantes comunitarios presentan las siguientes ventajas clave frente a los metálicos:

- Menor peso que los mismos en metal.

- Comportamiento excelente ante corrosión.

- Mayor facilidad de montaje al tener menor peso.

- Durabilidad.

- Menor rozamiento y menor conductividad

Por contrapartida podemos encontrar algunos inconvenientes como mayor coeficiente de dilatación que los realizados en materiales metálicos y el precio. 
A pesar de que inicialmente supongan un coste más elevado que los metálicos, por precio de material y por su montaje (soldaduras), no requieren de mantenimiento (repintado) ni problemas de oxidación y, técnicamente, al igual que los metálicos, son materiales que han demostrado ser aptos para su uso como colectores en hidrantes de riego.

Los resultados obtenidos en la Comunidad de Regantes de L'Alcúdia dónde se han instalado colectores plásticos son excelentes, al igual que en otras comunidades.

A la hora de instalar los elementos que componen un hidrante multiusuario, nuestro innovador "Tipo Costella" posee una ventaja sobre los hidrantes en " $\mathrm{T}$ " comúnmente instalados, la colocación de sus contadores de chorro múltiple en posición horizontal y el poder mantener las distancias rectas requeridas en los woltmann.

Como sabemos, los contadores de chorro múltiple, son los más utilizados en los hidrantes multiusuario y su posición óptima de instalación, horizontal. Una instalación inclinada o en vertical es muy habitual pero inadecuada, reducen la sensibilidad del contador a caudales bajos y perjudican la vida útil del mismo al apoyar la turbina sobre el eje de manera incorrecta.

El principal parámetro que afecta a la metrología de los contadores tipo woltmann es la distorsión del perfil de velocidades que se genera cuando no se mantienen las distancias rectas requeridas aguas arriba. En estos casos, el resultado de la medición es impredecible, y no se garantiza una mínima calidad de medida donde puede salir perjudicado tanto el usuario como el suministrador.

Otros aspectos innovadores a destacar de este nuevo hidrante multiusuario "Tipo Costella" son:

- Máxima maniobrabilidad en explotación, con una disposición compacta, que deja un amplio espacio de trabajo dentro de la caseta del hidrante (Figura 3).

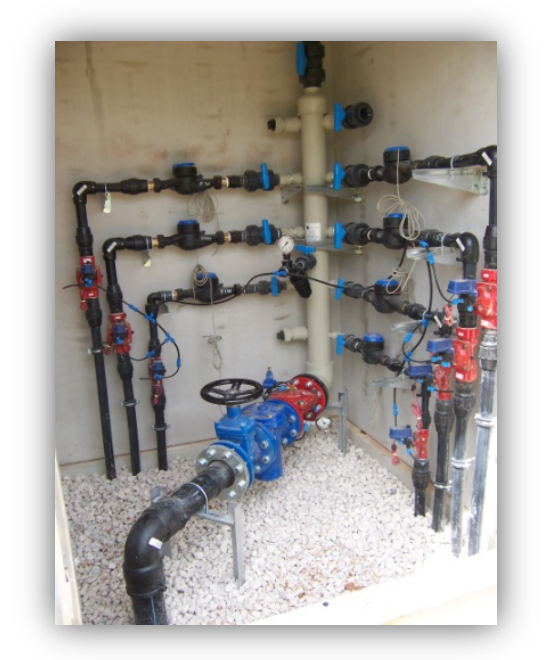

Figura 3. Hidrante instalado en la CCRR L'Alcúdia.

- Minimizar los robos y actos vandálicos, con la incorporación de una puerta metálica con perfiles de refuerzo, cerradura con tres puntos de anclaje y bisagras antivandálicas así como sensores de intrusión (Figura 4) y zumbido en el interior del hidrante avisando que ha 
sido detectado, los contadores de plástico incorporados también son disuasorios frente al robo.

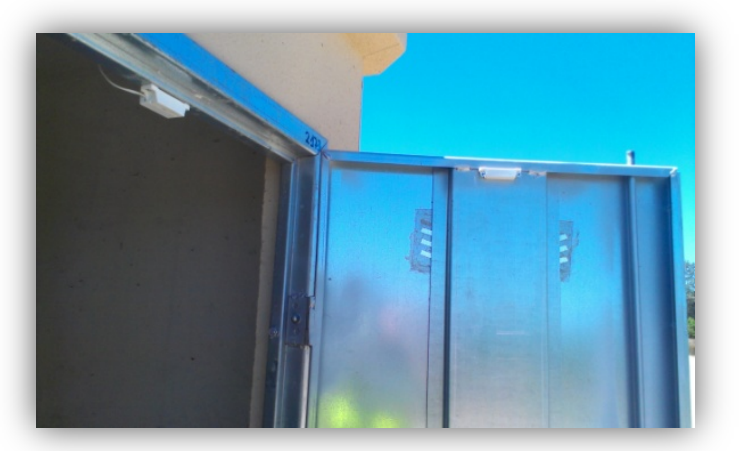

Figura 4. Sensor de intrusión y refuerzo con perfiles en puerta de hidrante.

\section{- Sistema de monitorización ambiental multipropósito}

A nivel práctico, en la Comunidad de Regantes de l'Alcúdia, se ha innovado en monitorización ambiental incorporando tres módulos de aplicación:

- Detección precoz de heladas.

- Compatibilización de Agricultura Ecológica.

- Control del perfil de humedad del suelo y riego eficiente.

Para la prevención temprana de heladas se han instalado sondas de temperatura y humedad relativa previamente calibradas en emplazamientos estratégicos obteniendo así los datos de las zonas más sensibles a heladas.

Los datos de temperatura y humedad relativa de estas sondas se leen cada 15 minutos enviándolos vía web al centro de control y procesándolos para:

- Obtención de gráficas de seguimiento.

- Gestión de alarmas.

Con el programa de monitorización ambiental implantado en la Comunidad de Regantes de L'Alcúdia controlamos la temperatura mínima a la que deseamos que se dispare la "alarma anti-heladas". Pueden programarse alarmas para cada punto de lectura de temperatura y, en función del tipo de sistema antihelada y las características de la red de riego, se configuran unas alarmas que actúan, de forma automática, sobre el programador de riego (telecontrol) activando las parcelas correspondientes.

Este sistema posee una doble función. Protección ante las heladas y poder controlar de forma contrastada las horas frío necesarias en algunas variedades de frutales de hueso, cultivando así nuevas variedades o cultivos que sin este dispositivo no serían posibles.

La Comunidad de Regantes dispone de un sensor de Temperatura y HR móvil que instalando en cualquier punto puede comprobar perfiles de temperatura a diferentes alturas y desde el software (figura 5) cambiar fácilmente el umbral de alarma que queremos programar para cada día. 


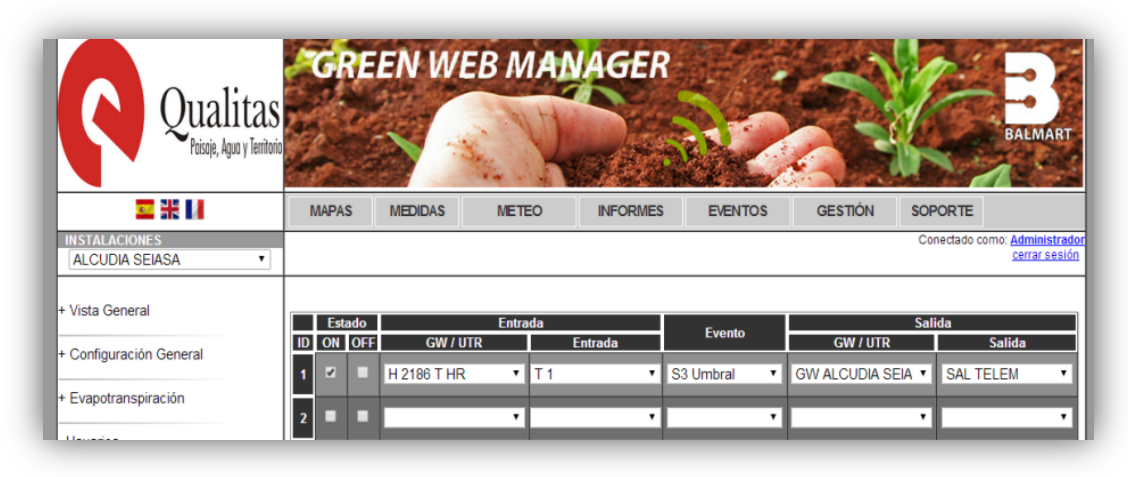

Figura 5. Software antiheladas.

En el ámbito de la Comunidad de Regantes del Sector II de L'Alcúdia existe una importante superficie dedicada al cultivo ecológico y por ello, se ha implantado un sistema de monitorización que permite controlar la presencia en el agua de riego de fertilizantes y otros productos permitidos en la agricultura tradicional, pero no en la agricultura ecológica, elaborando un Informe automáticamente emitido por la Comunidad de Regantes para los usuarios que soliciten necesaria la certificación de sus productos (GlobalGap, Naturane...).

Los dos cabezales de la red disponen de instalaciones de inyección de abono líquido y mediante un programa informático se ha conseguido una modelización hidráulica de la red (figura 6) para conocer, en cada momento, la concentración de producto y su distribución en la red de tuberías, es decir, se analiza el comportamiento hidráulico de la red desde el punto de vista de la calidad de las aguas conociendo el tiempo que tarda la red en limpiarse de agua mezclada con fertilizante para cualquier punto de la misma.

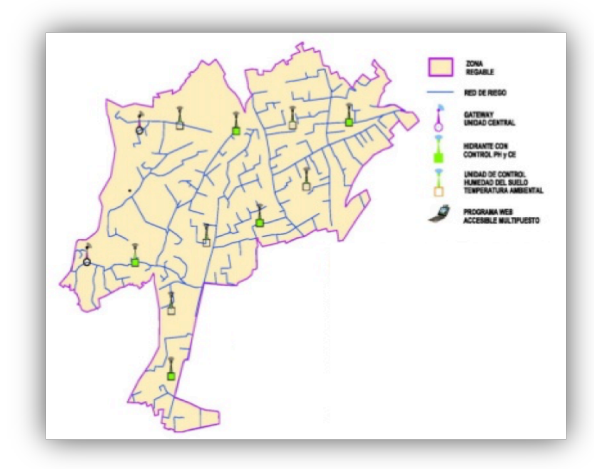

Figura 6. Modelización de la red.

Estos datos obtenidos mediante la modelización matemática se validan en campo gracias a la información de sensores de $\mathrm{pH}$ y conductividad eléctrica, parámetros de calidad del agua, distribuidos en la red de riego en cinco hidrantes testigo (figura 7) y en los cabezales. Las lecturas de $\mathrm{pH}$ y CE son tomadas cada $15 \circ 20$ minutos y transmitidas inalámbricamente de una estación concentradora a un servidor central vía GSM/GPRS. Este servidor central es un servidor web que permite el acceso por Internet para visualizar las medidas y estado de los sensores de manera instantánea. 


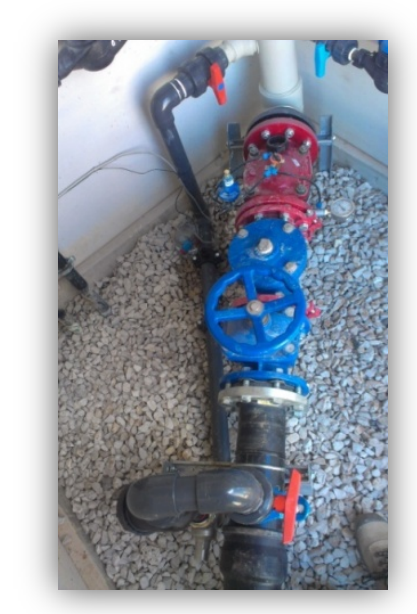

Figura 7. Sondas de $\mathrm{pH}$ y $\mathrm{CE}$ en hidrante

Este innovador sistema de monitorización ambiental cumple dos objetivos:

- Posibilidad de poder realizar Agricultura con Certificación Ecológica cumpliendo con todos los requisitos aplicables sin interferir en los intereses de la agricultura tradicional. Emisión de certificados consensuados con el CAECV.

- Asegurar que las concentraciones de fertilizantes que llegan a las parcelas de cultivo tradicional se realizan de forma adecuada y homogénea aportando abonados muy puntuales por cultivos o en diferentes momentos fenológicos de la planta mejorando así la calidad de los productos.

También se ha incorporado en la Comunidad de Regantes de L'Alcúdia sensores de medida de la temperatura y humedad relativa del aire, de la humedad del suelo (figura 8) y una estación meteorológica, permitiendo, con estos datos, una gestión más eficiente.

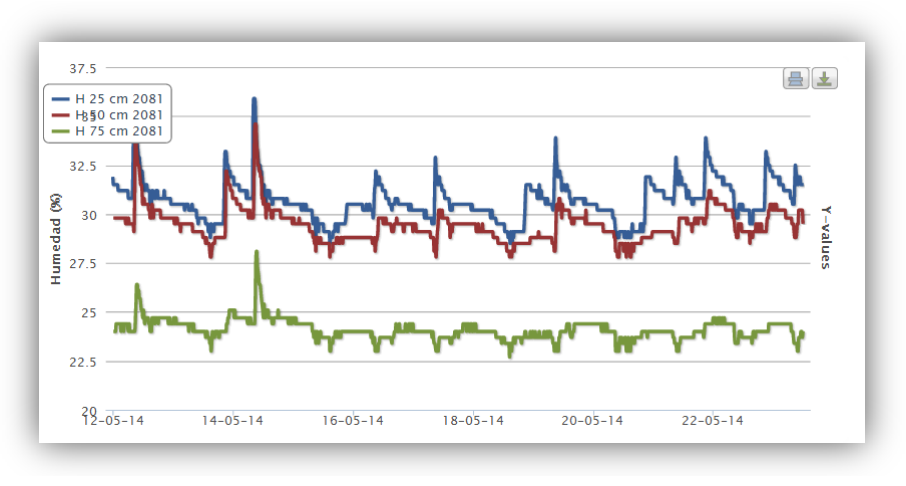

Figura 8. Frente húmedo a diferentes profundidades y tiempos de riego.

\section{- Mejora de la gestión integral hídrica y energética}

En los últimos tiempos ya nadie discute las ventajas que supone tener un Sistema de Información Geográfico (GIS o SIG), basadas en la asombrosa capacidad de gestión que se logra y la posibilidad de disponer e intercambiar información de cualquier tipo a través de medios como Internet.

Un SIG podría definirse de manera estándar como un complejo sistema de "hardware" y "software" que tiene como objeto la comprensión y análisis de datos espaciales 
georreferenciados cuyo fin último es ayudar a las diversas actividades humanas donde los datos espaciales tienen un papel determinante.

En la Comunidad de Regantes de L'Alcúdia se ha incorporado esta innovadora herramienta, un GIS y VISOR GIS (figura 9) con el fin de poder explotar la información gráficamente y gestionar todos los parámetros de la red en el mismo entorno.

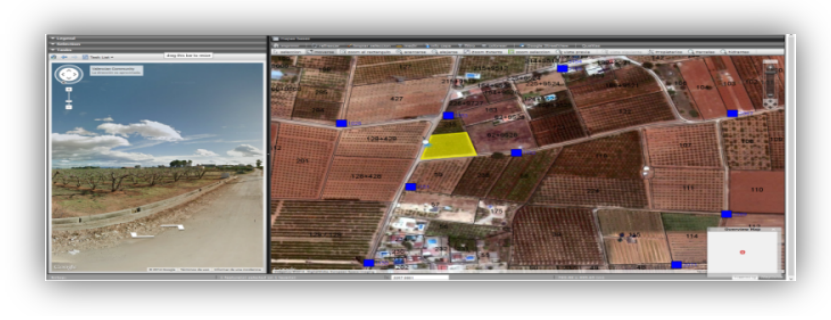

Figura 9. Visor GIS.

Todos los programas de la Comunidad de Regantes han quedado vinculados (uniendo telecontrol, catastro, sensórica, programa de mantenimiento....) introduciendo en el GIS todos los datos de la red ejecutada con una vista directa en el plano de datos (diámetro tuberías, materiales, arquetas, datos de propietario, etc.) facilitando así la gestión y explotación de los múltiples datos que tiene una Comunidad de Regantes.

Para una gestión que no implique estar delante del ordenador y que pueda visualizarse y manejarse desde cualquier punto se ha desarrollado su aplicación para móvil y tablet. Por tanto, se puede decir que llevamos en el teléfono o tablet los planos "de bolsillo". Se ha desarrollado una personalización completa a las necesidades e inquietudes de la Comunidad de Regantes.

El visor GIS (con acceso on line) se ha desarrollado para que el personal de la Comunidad de Regantes pueda visualizar de forma sencilla los datos contenidos en el GIS.

Se ha incorporado un programa de mantenimiento vinculado al GIS (figura 10) en el que se incluyen las tareas de mantenimiento preventivo y correctivo.

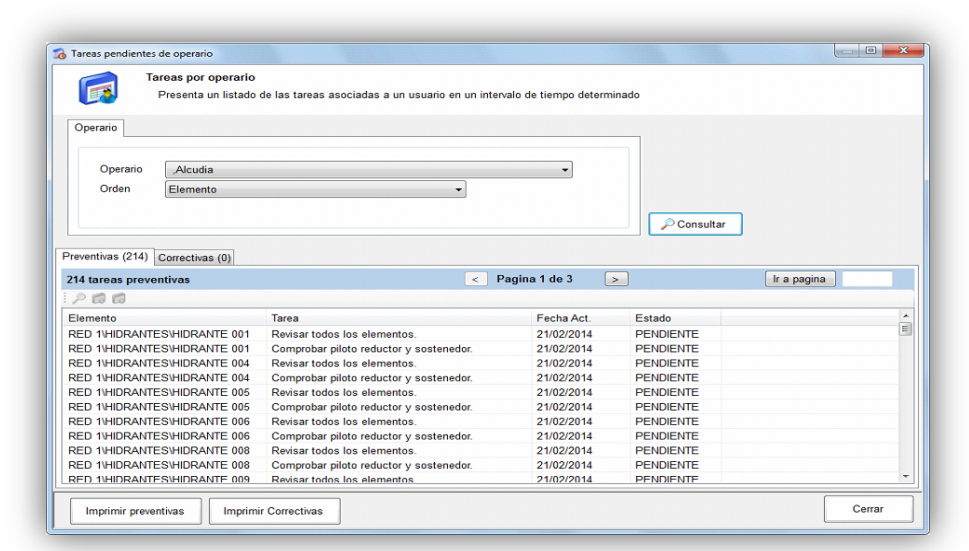

Figura 10. Programa de mantenimiento con aplicación para dispositivos móviles

Un requisito imprescindible para la consecución de una alta eficiencia en el riego, a parte de un diseño óptimo de las instalaciones y una ejecución adecuada, es una buena gestión en la etapa de explotación. Por ello, afirmamos, que un sistema GIS aplicado a una 
comunidad de regantes es una de las mejores innovaciones tecnológicas que se pueden implantar, siendo esencial para una buena gestión integral hídrica.

Otros aspectos incorporados en L'Alcúdia dentro del área de mejora de la gestión integral hídrica y energética son:

- Analizadores de red con fin de controlar los parámetros necesarios para la optimización energética de la Comunidad de Regantes minimizando sus costes. Asimismo se da prioridad a las soluciones técnicas que minimicen el consumo de agua presurizada.

- Dos bypass de la red 1 a la red 2. En el proyecto original las dos redes eran independientes y con estos bypass hemos conseguido, por un lado, poder abastecerse de una red a otra en caso de rotura o problema en alguna de las redes y por otro, poder llenar depósito de cota desde una de las redes en horas más económicas y regar desde dicho cabezal a ambas redes durante el día, minimizando costes.

- Limitadores de caudal en las parcelas que poseen balsas propias con el fin de evitar desestabilización de caudales de la red y un eficiente consumo de agua.

\section{- Mejora medioambiental}

Se han instalado 10 bocas contraincendios en los hidrantes (figura 11) consensuando su ubicación con el agente medioambiental de la zona y cuya geolocalización es proporcionada a la brigada responsable de extinción de incendios, mejorando con ello la lucha contra incendios.

Se han incorporado 8 surtidores de toma (figura 11) facilitando el llenado de cubas para tratamientos fitosanitarios de los usuarios de la Comunidad de Regantes, con la eliminación de la posible contaminación en las recargas.

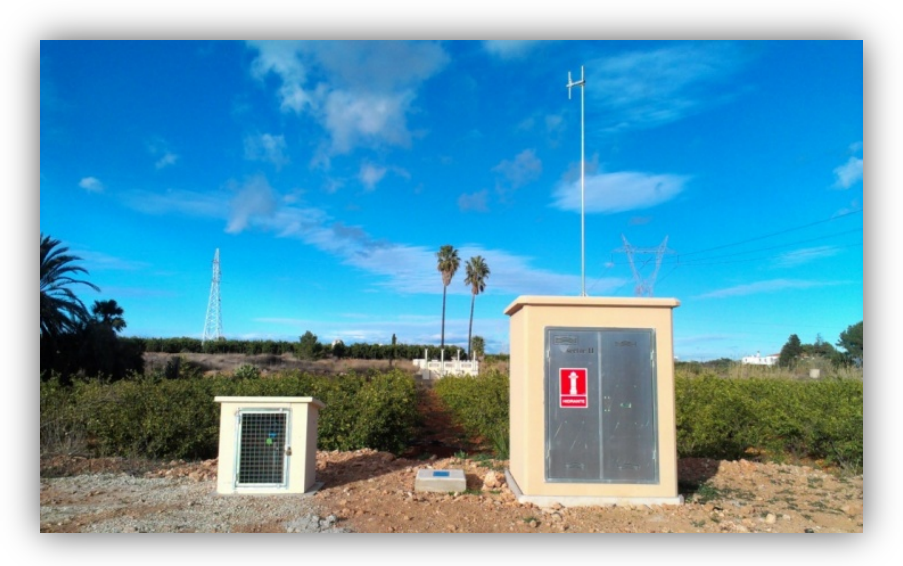

Figura 11. Boca de incendio y surtidor de toma.

Con el fin de evitar problemas de rotura de depósitos de fertilizantes y poder generar contaminación de suelo y aguas subterráneas se han construido unos cubetos de contención de fertilizantes.

Se ha realizado experiencia piloto de reservorios biológicos empleando las ubicaciones de las casetas de hidrante multiusuario con disponibilidad de agua para su implantación. Se han realizado plantaciones con especies vegetales en las inmediaciones como hábitat alternativo para la entomofauna auxiliar y pueden contribuir al control biológico de plagas agrícolas y disminución de fitosanitarios. 
- Mejora de calidad de las aguas y seguridad en abastecimiento hídrico en las CCRR en periodos de sequía

En cuanto a la mejora de calidad de las aguas se ha establecido un sistema antialgas mediante ultrasonidos para el depósito Majada de las Cabras.

El depósito de Florentina se ha cubierto con una doble malla de rafia (figura 12) provocando una reducción de la evaporación y por tanto un ahorro hídrico considerable. Se mejora el mantenimiento del depósito y de los elementos instalados en el cabezal debido a menores concentraciones de suciedad y elementos arrastrados por el viento dentro del depósito. La cubrición del depósito también ayuda a la no proliferación de algas.

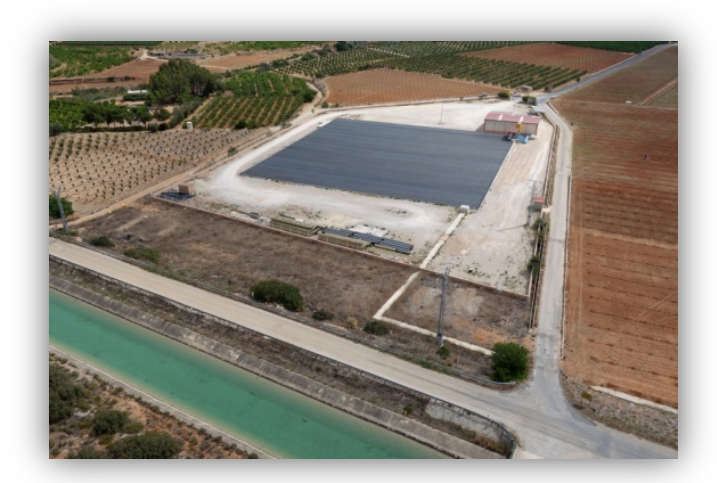

Figura 12. Rafia doble malla, depósito Florentina.

Para tener una seguridad en el suministro hídrico en momentos en los que la Comunidad de Regantes posea restricciones hídricas en el Canal Júcar Turia por motivos derivados de la sequía, se ha ejecutado un cabezal de inyección desde tres pozos gestionados por la misma comunidad.

\section{3- Conclusiones y recomendaciones}

En cuanto a las recomendaciones indicar:

- En fase de proyecto se han de incorporan todas las medidas posibles como las expuestas que permiten a la entrega de la obra una perfecta gestión y conservación de las mismas.

- Dar la importancia que se requiere a los cultivos de la zona regable para incidir en aquellas soluciones que mejoran producciones.

Como conclusiones:

- La modernización debe repercutir en beneficio de la sociedad. Por ello todas aquellas medidas que hagan un medioambiente más sostenible harán que se perciba estas obras como positivas no solo para el sector agrario.

- Hay que proporcionar a las Comunidades de Regantes las herramientas que permitan dar un servicio a sus comuneros de excelencia. No solo de la cantidad de agua que tengan concedida, sino con la distribución en el tiempo y en horarios que se tenga la garantía de optimizar las cosechas.

- A modo de conclusión final exponemos que el coste final de las medias relacionadas ha supuesto un $4 \%$ del coste total de las obras. Y ya solo en el 
primer año de explotación se han evitado heladas, se han mantenido las certificaciones ecológicas, no se han producido robos, y se tiene la constancia de modo fehaciente de un incremento de cosechas equivalente al $9 \%$. Que sobre la superficie regada ha supuesto cerca de $250.000 \mathrm{~kg}$ de la mejor calidad de fruta.

\section{4- Agradecimientos}

Agradecer la estrecha colaboración entre todos los integrantes de la obra, especialmente a la Comunidad de Regantes de L'Alcudia con los cuales ha sido posible la implantación de las innovaciones descritas en el presente documento. Asimismo agradecer a todos los Organismos e Instituciones que han posibilitado su ejecución y financiación. 